\title{
Three Combined Quantitative Trait Loci from Nonhost Lactuca saligna Are Sufficient to Provide Complete Resistance of Lettuce Against Bremia lactucae
}

\author{
Ningwen W. Zhang, Koen Pelgrom, Rients E. Niks, Richard G. F. Visser, and Marieke J. W. Jeuken \\ Laboratory of Plant Breeding, Wageningen University, PO Box 386, 6700 AJ, Wageningen, The Netherlands \\ Submitted 30 January 2009. Accepted 23 April 2009.
}

\begin{abstract}
The nonhost resistance of wild lettuce (Lactuca saligna) to downy mildew (Bremia lactucae) is based on at least 15 quantitative trait loci (QTL), each effective at one or more plant developmental stages. We used QTL pyramiding (stacking) to determine how many of these QTL from $L$. saligna are sufficient to impart complete resistance towards $B$. lactucae to cultivated lettuce, $L$. sativa. The alleles of four of the most promising QTL, rbq4, rbq5, rbq6+11, and $r b q 7$ are effective at both the young and adult plant stages. Lines with these four QTL in all possible combinations were generated by crossing the respective backcross inbred lines (BIL). Using the 11 resulting lines (combiBIL), we determined that combinations of three QTL, rbq4, rbq5, and $r b q 6+11$, led to increased levels of resistance; however, one QTL, rbq7, did not add to the resistance level when combined with the other QTL. One line, tripleBIL268, which contains the three QTL $r b q 4, r b q 5$, and $r b q 6+11$, was completely resistant to $B$. lactucae at the young plant stage. This suggests that these three QTL are sufficient to confer the complete resistance of the nonhost $L$. saligna and any additional QTL in $L$. saligna are redundant. Histological analysis of $B$. lactucae infection in $L$. saligna, the BIL, and the combiBIL $48 \mathrm{~h}$ after inoculation revealed different microscopical phenotypes of resistance. The QTL differed with respect to the stage of the infection process with which they interfered.
\end{abstract}

Nonhost resistance is the most common form of resistance in a given plant species to provide both strong and broad protection against potential pathogens (Heath 1981; Niks 1988). Compared with host resistance, the mechanisms underlying nonhost resistance are not as well understood (Mysore and Ryu 2004; Schweizer 2007). The investigation of the genetic inheritance of nonhost resistance will lead to the identification of genes that determine whether a plant is a host or nonhost to a particular pathogen. However, it is difficult to study the genetics of nonhost resistance, as host and nonhost plant species are usually either not crossable or give aberrant or sterile progeny when crossed (Atienza et al. 2004; Jeuken and Lindhout 2002; Niks 1988). In the lettuce (Lactuca spp.)-downy mildew (Bre-

Corresponding author: N. Zhang; Telephone: +0031-317-482835; Fax: +0031-317-483457; E-mail: ningwen.zhang@wur.nl

* The $\boldsymbol{e}$-Xtra logo stands for "electronic extra" and indicates that Figure 3 appears in color online. mia lactucae) plant pathosystem, the nonhost species L. saligna is crossable with the host species L. sativa. This makes it possible to study the inheritance of nonhost resistance.

We were able to identify 15 backcross inbred lines (BIL) displaying race-nonspecific quantitative resistance at one or more plant developmental stages (Zhang et al. in press) by infecting a set of 28 BIL covering $94 \%$ of the L. saligna genome. Each BIL contains one introgression of L. saligna in $L$. sativa background (Jeuken and Lindhout 2004; Jeuken et al. 2008). The 15 BIL introgressions are considered to represent 15 quantitative trait loci (QTL), but it is possible that some introgressions contain more than one gene that contributes to quantitative resistance. QTL were detected at different developmental stages of lettuce plants by seedling disease tests (SDT; $n=5$ ), young plant disease tests (YDT; $n=11$ ), adult plant disease tests in the greenhouse $\left(\mathrm{ADT}_{\mathrm{G}} ; n=6\right)$, and adult plant disease tests in the field $\left(\mathrm{ADT}_{\mathrm{F}} ; n=9\right)$ (Zhang et al. in press). These QTL displayed relative infection severities (RIS) of 10 to $79 \%$ compared with the $100 \%$ displayed by the susceptible parental line L. sativa cv. Olof. These findings suggest that the nonhost resistance of L. saligna is based on at least 15 QTL, each with moderate to large effect (Zhang et al. in press).

Exploiting this nonhost resistance in cultivated lettuce might be beneficial, since the resistance might prove more durable than the hitherto extensively deployed $\mathrm{Dm}$ genes (Lebeda and Schwinn 1994). In order to exploit this nonhost resistance in cultivated lettuce ( $L$. sativa), we need to know how many of the about 15 QTL are sufficient to give $L$. sativa complete protection to $B$. lactucae. One way to address this question is to pyramid (stack) different QTL in the L. sativa background and evaluate their effects on infection severity. However, it is almost impossible to develop all of the thousands of different combinations of two to 15 QTL. Therefore, we selected four recessive QTL, $r b q 4, r b q 5, r b q 6$, and $r b q 7$, that are effective at both the young and the adult plant stages and combined them into L. sativa cv. Olof background (Jeuken et al. 2008; Zhang et al. in press). These QTL were mapped in the L. saligna introgressions of BIL8.2, BIL2.2, BIL6.3, and BIL4.2, respectively. In BIL6.3, the presence of $r b q 6$ is always combined with another QTL, Rbq11, which is only effective at the young plant stage and is very likely to be located at 0 to $16 \mathrm{cM}$ of the 32-cM-long introgression on the top of chromosome 4 (Zhang et al. in press). We refer to this situation as $r b q 6+11$, and count them as one inseparable QTL. The QTL regions for $r b q 4$, $r b q 5, r b q 6+11$, and $r b q 7$ do not overlap with known clusters of $\mathrm{Dm}$ resistance genes at chromosomes 1, 2, 4, and 8, accord- 
ing to Jeuken and associates (2008). To study the interactions between these selected QTL, eleven lines that combined different combinations of homozygous $L$. saligna introgressions carrying the respective QTL alleles in the L. sativa background were developed and designated as combiBIL.

In most biotrophic plant pathosystems, the mechanism of resistance to incompatible (nonhost) pathogens differs from qualitative resistance in a host to its compatible pathogen species. The latter case is usually based on posthaustorial hypersensitive response (HR) (Niks and Dekens 1991; Niks and Rubiales 2002), while nonhost resistance is often characterized by prepenetration defense. Most examples of such mechanisms involve fungal biotrophic pathogens, especially powdery mildews and rusts (Ferreira et al. 2006; Heath 2000, 2001; Niks and Rubiales 2002). In oomycetes, HR seems to play a comparatively larger role, as it is associated with both resistance gene-mediated and nonhost resistance (Kamoun et al. 1999; Vleeshouwers et al. 2000).

We carried out histological observations to characterize microscopical responses of the target QTL on the development of B. lactucae, an obligate biotrophic oomycete pathogen. The present histological study might reveal indications about the resistance mechanisms. Different microscopical responses of resistance in accessions of Lactuca spp. to B. lactucae have been described, including differences in the timing and rate of pathogen infection structure development, the occurrence of HR (Lebeda et al. 2001, 2002; Maclean and Tommerup 1979), accumulation of phenolics (Bennett et al. 1996), and occurrence of oxidative stress (Bestwick et al. 2001).

The microscopical responses of nonhost resistance of $L$. saligna accessions seem to be very different from race-specific resistance conferred by $D m$ genes in lettuce cultivars and from the partial (field) resistance of lettuce cv. Iceberg (Lebeda and Reinink 1994). At 48 h postinoculation (hpi), $L$. saligna CGN05271 had a much lower frequency of hypha and haustorium formation but a higher frequency of epidermal necrosis at the infection sites compared with susceptible $L$. sativa cultivars (Lebeda and Reinink 1994). Some studies have reported variation between $L$. saligna accessions in their predominant microscopical responses of resistance to B. lactucae (Lebeda and Reinink 1994; Lebeda et al. 2006; Sedlářová et al. 2001).

The present study reports on i) the development of eleven combiBIL, ii) the comparison of resistance levels between the eleven combiBIL and the BIL carrying individual resistance QTL, primarily at the young plant stage, iii) determination of interactions between QTL, and iv) the microscopic responses of $L$. saligna and the effects of the QTL alleles on the development of B. lactucae based on histological studies.

\section{RESULTS}

\section{Development of eleven combiBIL.}

The BIL with the relevant introgressions were crossed to obtain an $\mathrm{F}_{2}$ in which, by marker assisted selection (MAS) using the expressed sequence tag (EST) markers at the introgression regions, the homozygous doubleBIL26 and doubleBIL48 were selected (Fig. 1). Next, these doubleBIL were crossed and their $\mathrm{F}_{2}(n=48)$ were screened using EST markers. This resulted in the selection of doubleBIL24 and six plants with potential genotypes that could lead to the other eight types of combiBIL (three doubleBIL, four tripleBIL, and one quartoBIL). These six plants were selfed to obtain the $\mathrm{F}_{3}$ and $\mathrm{F}_{4}$ generations (Fig. 1). After genotyping the $F_{3}$ and the $F_{4}$ plants, the other eight combiBIL were selected by MAS and genotypes of all eleven combiBIL were confirmed using 94 molecular markers scattered across the introgression segments.
Due to the extra introgression on top of chromosome 4 (0 to $32 \mathrm{cM}$ ) in BIL6.3, all the combiBIL that were derived from BIL6.3 also had this introgression. Severe preference of $L$. saligna alleles on the top of chromosome 4 prevented the selection of genotypes without this introgression. Therefore, $r b q 6$ and Rbq11 were always combined in each combiBIL derived from BIL6.3. DoubleBIL28 contained a small introgression from 29 to $43 \mathrm{cM}$ on chromosome 4 derived from the introgressed segment of BIL4.2 (Fig. 1), but this doubleBIL may not contain either Rbq11 (located on segment 4.1) or rbq7 (located on segment 4.2) for two reasons: i) Rbql1 is likely to locate on the top of chromosome 4 from 0 to $16 \mathrm{cM}$ (Zhang et al. in press) and ii) BIL4.2 introgression is nearly $60 \mathrm{cM}$ (29 to $83 \mathrm{cM})$ long and the chance of $r b q 7$ residing in 29 to $43 \mathrm{cM}$ is low.

Introgression segments in BIL2.2, BIL6.3, and BIL8.2, in which $r b q 5, r b q 6+11$, and $r b q 4$ reside, caused a morphology that only slightly deviated from L. sativa cv. Olof. The introgression in BIL4.2 ( $r b q 7)$ caused a more severely deviated morphology with a dark-green, long, narrow leaf and nonheading (Jeuken and Lindhout 2004). The same morphological features were logically pyramided in the subsequent combiBIL.

CombiBIL containing introgressions of BIL6.3 $(r b q 6+11)$ and BIL8.2 ( $r b q 4)$ were expected to be difficult to retrieve due to severely distorted segregations with a deficit of homozygous L. saligna genotypes in the $\mathrm{F}_{2}$ population (Jeuken et al. 2001, 2008). The segregation ratios of introgression 6.3 and 8.2 during combiBIL development are listed in Table 1. Surprisingly, the segregation of introgression 6.3 was not as distorted as observed in the original $\mathrm{F}_{2}$ population from L. saligna $\mathrm{CGN} 05271 \times L$. sativa cv. Olof $(n=126)$. The allele frequency of $L$. saligna was 0.46 and the genotype frequency of homozygous $L$. saligna was 0.26 , close to the respective expected values of 0.5 and 0.25 . The segregation of introgression 8.2 deviated significantly from an expected ratio of 1:2:1 (Chi-square test, $P<0.001$ ) because of a clear deficit of homozygous $L$. saligna genotypes and excess of

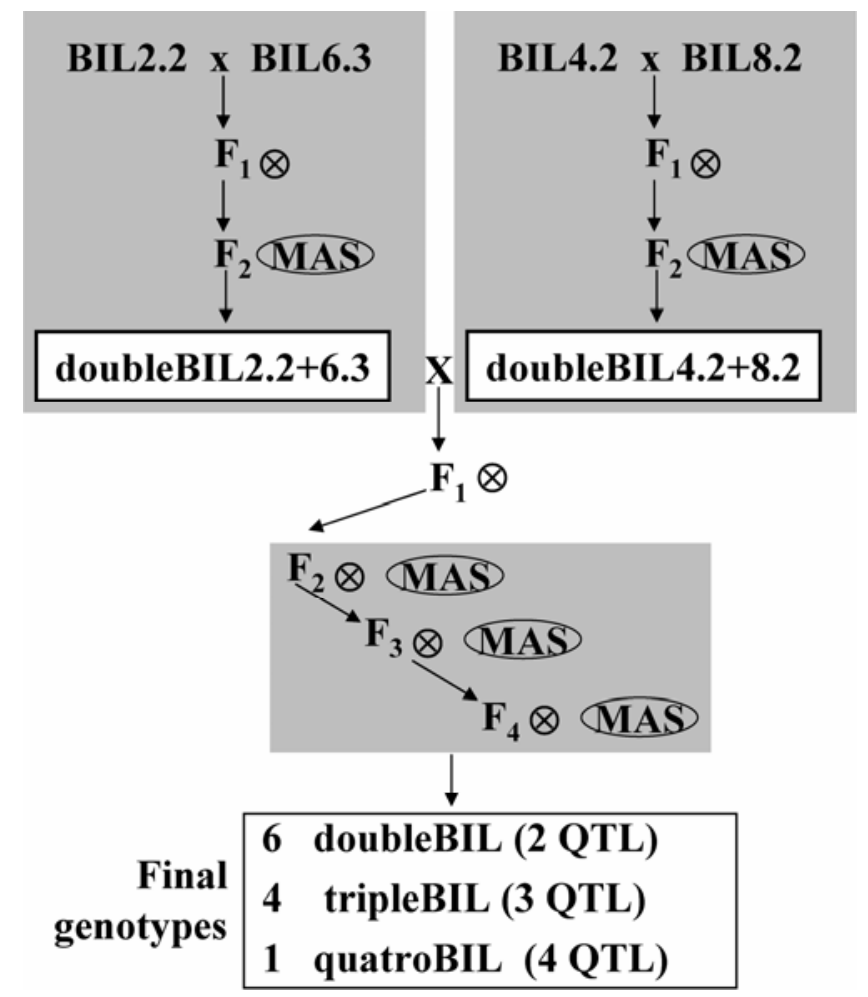

Fig. 1. Development of the lettuce downy mildew resistance quantitative trait loci-pyramided population. MAS $=$ marker assisted selection. $\otimes$ indicates selfing. 
heterozygous genotypes, while the allele frequency stayed normal for the L. saligna allele (Table 1).

\section{Disease evaluation for combiBIL.}

We first evaluated the infection severities of the six doubleBIL compared with the four individual BIL using the four different types of bioassays. In subsequent experiments, all 11 combiBIL (quatro-, triple-, and doubleBIL) were evaluated only at the young plant stage, due to time and space limitations (Fig. 2).

The results are presented as RIS compared with the susceptible parental line L. sativa cv. Olof as described below. Therefore, L. sativa cv. Olof was always set as $100 \%$ RIS, although it showed 59, 83, and $60 \%$ absolute infection severity at the seedling (SDT), young plant (YDT), and adult plant $\left(\mathrm{ADT}_{\mathrm{G}}\right)$ stages, respectively, and scored 7 on a 0 to 10 scale (resistant to susceptible) in the field test $\left(\mathrm{ADT}_{\mathrm{F}}\right)$. L. saligna showed $0 \%$ infection severity in all the experiments. The additional susceptible reference lines, BIL4.4, BIL5.1, and BIL8.1 all showed either a similar or higher infection severity (90 to $153 \%$ ) compared with $L$. sativa cv. Olof. All RIS values for the target BIL were as expected and in accordance with earlier results at all developmental stages (Fig. 2) (Zhang et al. in press). In summary, BIL2.2 and BIL4.2 showed quantitative resistance at all four developmental stages; BIL8.2 was resistant in the YDT, $\mathrm{ADT}_{\mathrm{G}}$, and $\mathrm{ADT}_{\mathrm{F}}$ and BIL6.3 in the YDT and $\mathrm{ADT}_{\mathrm{G}}$. BIL4.1 showed quantitative resistance only in the YDT.

To describe the phenotypic effects of interactions of multiple loci (QTL allele combinations), we introduce the terms "epistatic interaction with a positive effect" and "epistatic interaction with a negative effect". Epistatic interaction is used if the size of the effect of a QTL allele depends on the presence of another resistance QTL allele. Such an interaction can lead to the combined effect of the QTL being significantly smaller (negative effect) or larger (positive effect) than that of the QTL allele with the largest individual effect. "No epistatic interaction" is used when the effect of the multiple resistance QTL alleles (in a combiBIL) is not significantly different from that of the individual resistance QTL alleles (in a BIL).

From the QTL combinations in the six doubleBIL, we observed three epistatic interactions with positive effects (doubleBIL68 in YDT and ADT $_{\mathrm{G}}$ and doubleBIL26 in YDT) and one epistatic interaction with a negative effect (doubleBIL46 in SDT) (Fig. 2). The results from the other QTL combinations in the six doubleBIL did not indicate an epistatic interaction between QTL.

At the seedling stage (SDT), the RIS of doubleBIL26, doubleBIL28, doubleBIL24, and doubleBIL48 were similar to their corresponding individual BIL, indicating no epistatic interactions between these QTL at this particular developmental stage (Fig. 2). As expected, doubleBIL68 showed susceptibility with similar RIS to L. sativa cv. Olof, since both BIL6.3 and BIL8.2 are susceptible at the seedling stage (Zhang et al. in press) (Fig. 2). Surprisingly, doubleBIL46 was susceptible, although we expected it would have a similar or lower RIS than BIL4.2 (rbq7) (Fig. 2). This suggested an epistatic interaction with a negative effect at the seedling stage when $r b q 6+11$ and rbq7 were combined.

At the adult plant stage in the greenhouse $\left(\mathrm{ADT}_{\mathrm{G}}\right)$, all doubleBIL had a significantly lower RIS (10 to 50\%) than $L$. sativa cv. Olof. Only doubleBIL68 showed a significantly lower RIS (10\%) than its corresponding individual BIL (BIL6.3, $38 \%$ RIS and BIL8.2, 28\% RIS), which suggested an epistatic interaction with a positive effect of alleles of QTL $r b q 6+11$ and $r b q 4$, leading to an increased level of resistance. The RIS of the other five doubleBIL were close to the levels of their respective individual BIL, indicating no epistatic interactions between these QTL (Fig. 2). In the field disease test (ADT ${ }_{\mathrm{F}}$ ), similar results were obtained as in the greenhouse, except that doubleBIL68 and doubleBIL28 had a lower (but not significantly so) RIS than their corresponding individual BIL (Fig. 2).

At the young plant stage (YDT), the correlation between the two experiments with only doubleBIL and the two with all combiBIL was high $(r=0.9)$. Therefore, we only present the results from the latter of these (Fig. 2). All the 11 combiBIL showed a significantly lower RIS than the susceptible $L$. sativa cv. Olof. Two doubleBIL, doubleBIL68 $(0.2 \%)$ and doubleBIL26 $(0.3 \%)$, stood out and showed very little sporulation. The RIS of these combiBIL was significantly lower than that of the corresponding individual BIL, indicating epistatic interactions with positive effects between these QTL (Fig. 2). DoubleBIL28 also had a low RIS (3\%), but it was not significantly lower than that of BIL2.2 (12\%).

TripleBIL268 showed complete resistance (0\% RIS) to $B$. lactucae, similar to the nonhost $L$. saligna. The corresponding doubleBIL (doubleBIL68, doubleBIL28, and doubleBIL26) all showed very little infection severity but were not completely resistant. These results validated the epistatic interactions with positive effects of $r b q 4, r b q 5$, and $r b q 6+11$ alleles for the resistance. The other three tripleBIL, tripleBIL246, tripleBIL248, and tripleBIL468, showed very low RIS (0.1 to 6\%) that were significantly lower than the corresponding individual BIL but not significantly lower than the corresponding doubleBIL (Fig. 2). For instance, tripleBIL468 (0.4\%) showed a similar severity level as doubleBIL68 $(0.2 \%)$. These three tripleBIL have introgression 4.2 in common, which showed that the resistance allele of $r b q 7$ did not contribute to a higher resistance level when combined with the other QTL alleles. QuatroBIL2468 also showed complete resistance like tripleBIL268 (Fig. 2).

\section{Histological observation.}

Macroscopic infection severity observations (RIS) of the examined genotypes (Table 2, RIS\% column) showed similar results as the previous disease tests at the young plant stage (Fig. 2). In this set of histological observations and in all tested lines, nonpenetrating appressoria were very rarely observed. Therefore, we excluded the frequency of appressoria formation from this report.

At 48 hpi with $B$. lactucae race $\mathrm{Bl}: 14$, the susceptible genotypes $L$. sativa cv. Olof and BIL4.4 showed a very high frequency $(>90 \%)$ of secondary vesicle $(\mathrm{SV})$ formation $(\mathrm{SV} / \mathrm{pri}$ -

Table 1. The segregation ratio, genotype frequency, and allele frequency of BIL6.3 $(r b q 6+11)$ and BIL8.2 $(r b q 4)$ introgression segments

\begin{tabular}{|c|c|c|c|c|c|c|}
\hline \multirow[b]{2}{*}{ Introgression } & \multicolumn{2}{|c|}{ Segregation ratio $(\mathbf{a} / \mathbf{h} / \mathbf{b})^{\mathbf{a}}$} & \multicolumn{2}{|c|}{ Genotype frequency of Lactuca saligna } & \multicolumn{2}{|c|}{ Allele frequency of $L$. saligna } \\
\hline & $\mathbf{F}_{2}$ & BIL & $\mathbf{F}_{2}$ & BIL & $\mathbf{F}_{2}$ & BIL \\
\hline BIL6.3 & $36: 46: 9(P<0.001)$ & $60: 68: 45(P<0.005)^{\mathrm{b}}$ & 0.10 & 0.26 & 0.35 & 0.46 \\
\hline BIL8.2 & 30:90:6 $(P<0.001$ & $192: 228: 8(P<0.001)^{\mathrm{c}}$ & 0.05 & 0.02 & 0.40 & 0.55 \\
\hline
\end{tabular}


mary vesicle $[\mathrm{PV}]$ ), hyphae (HY) formation (HY/SV), and haustoria (HA) formation (HY/HA), and an HR frequency of 56 and $43 \%$, respectively. The HR was mostly restricted to the epidermal cell, in which the penetration took place (Table 2; Figs. 3 and 4).

The most striking observations for the nonhost L. saligna compared with $L$. sativa cv. Olof were that no real healthy hyphae were observed (consequently the frequency of haustorium was $0 \%$ ) and $23 \%$ of SV formed sort of malformed hypha-like structures (mal-HY) (Fig. 3). The mal-HY and $0 \%$ real hyphae formation suggested that all the infection units
(IU) in L. saligna were arrested before normal hypha formation. In addition, a higher frequency of HR appeared in $L$. saligna $(88 \%)$ than in L. sativa cv. Olof $(56 \%)$ (Table 2$)$. The resistance response of $L$. saligna seemed to be based mainly on prehyphal resistance and a high frequency of HR (Table 2; Figs. 3 and 4).

The B. lactucae development process in BIL2.2, BIL8.2, and BIL4.1 showed similar patterns to each other in the frequency of formation of each pathogen structure, in which the responses were intermediate between the susceptible and resistant parental lines. In these BIL, the infection was largely but

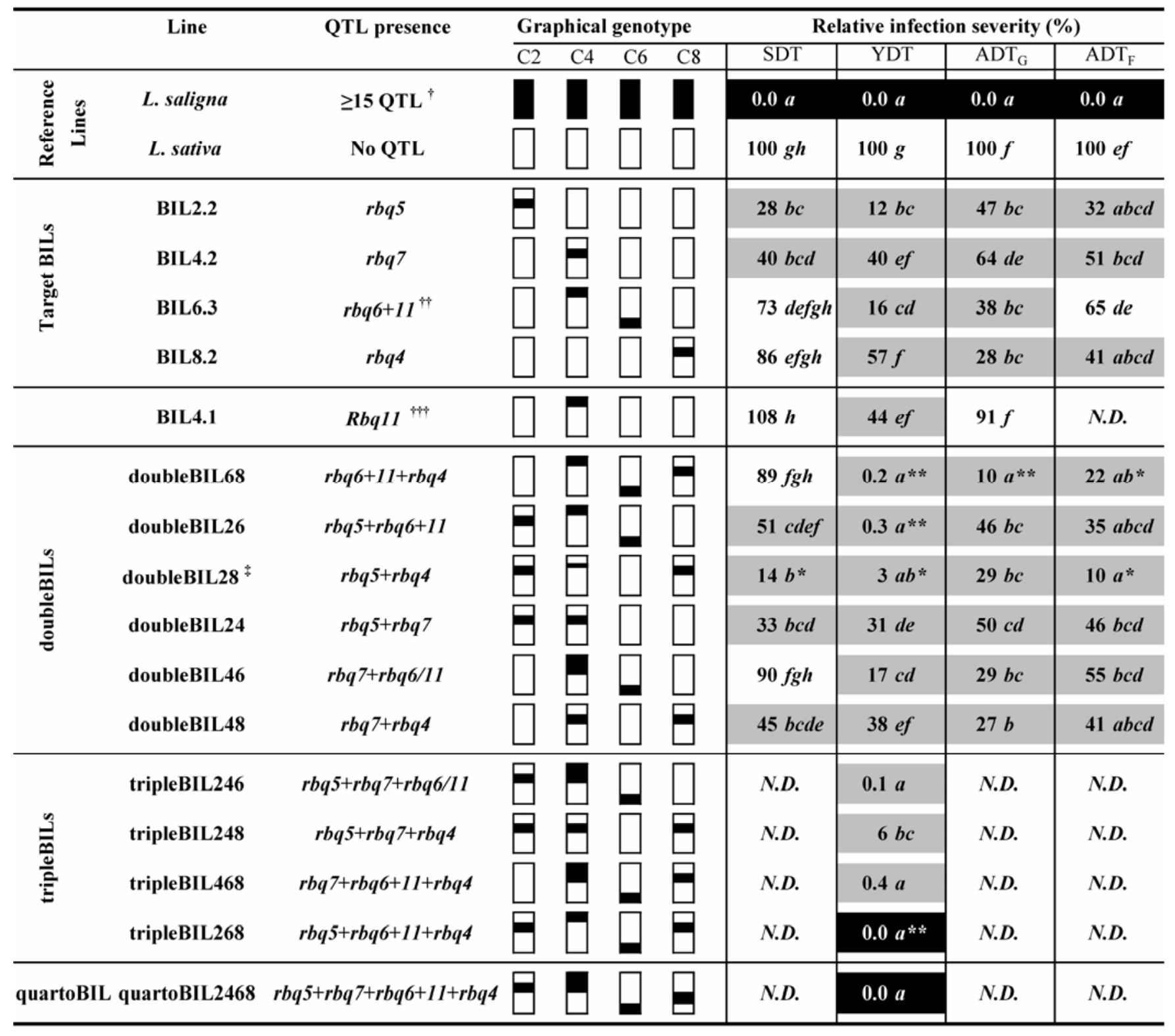

Fig. 2. The genotypes of the lettuce downy mildew resistance quantitative trait loci (QTL) pyramid population and their relative infection severities (RIS) to Bremia lactucae at various plant development stages. In the genotype presentation, C $x$ stands for chromosome $x$. A solid bar indicates the homozygous Lactuca saligna introgression; an open bar indicates the homozygous $L$. sativa background. For RIS compared with that of $L$. sativa cv. Olof, the absolute infection severity of L. sativa cv. Olof in seedling disease, young plant disease, adult plant disease in greenhouse, and adult plant disease in the field tests (SDT, YDT, $\mathrm{ADT}_{\mathrm{G}}$, and $\mathrm{ADT}_{\mathrm{F}}$, respectively) were $82,57,87$, and $25 \%$, respectively. Letters next to the RIS numbers indicate the significance between different lines within one type of disease test. Letters in common means no significant difference $(\alpha=0.05$, Tukey honestly significant differences test). Black grids indicate the completely resistant lines in the corresponding bioassay types; gray grids indicate the quantitatively resistant lines (significantly lower infection severity than cv. Olof with $95 \%$ confidence); white grids indicate the susceptible lines (not significantly different from $\mathrm{cv}$. Olof). N.D. $=$ not determined. BIL $=$ backcross inbred lines. $\dagger 15$ QTL are identified in $L$. saligna. $\dagger \dagger$ The presence of both $r b q 6$ and $R b q 11$ is designated $r b q 6+11$. $\dagger \dagger \dagger R b q 11$ is only effective at the young plant stage (Zhang et. al. in press). $\neq$ DoubleBIL28 contains an introgression on chromosome 4 that has a small overlap with BIL4.1 and BIL4.2, but the precise locations of Rbq11 and $r b q 7$ are unknown. * No significant difference from the individual BIL, but infection severity decreased suggestively than the individual BIL. **Lines showed an epistatic interaction of multiple QTL with significantly decreased infection severity compared to the individual BIL. 
not completely arrested before HY formation, since the HY/SV ratios in these lines were significantly lower than in the susceptible parent L. sativa cv. Olof (Table 2; Fig. 3). Low frequencies of mal-HY and normal HY with HA were observed. All three BIL had a higher proportion of subepidermal-HR (SEHR) occurrence, in contrast to $L$. sativa cv. Olof and $L$. saligna, which were mainly associated with epidermal-HR (EHR) (Table 2; Fig. 4).

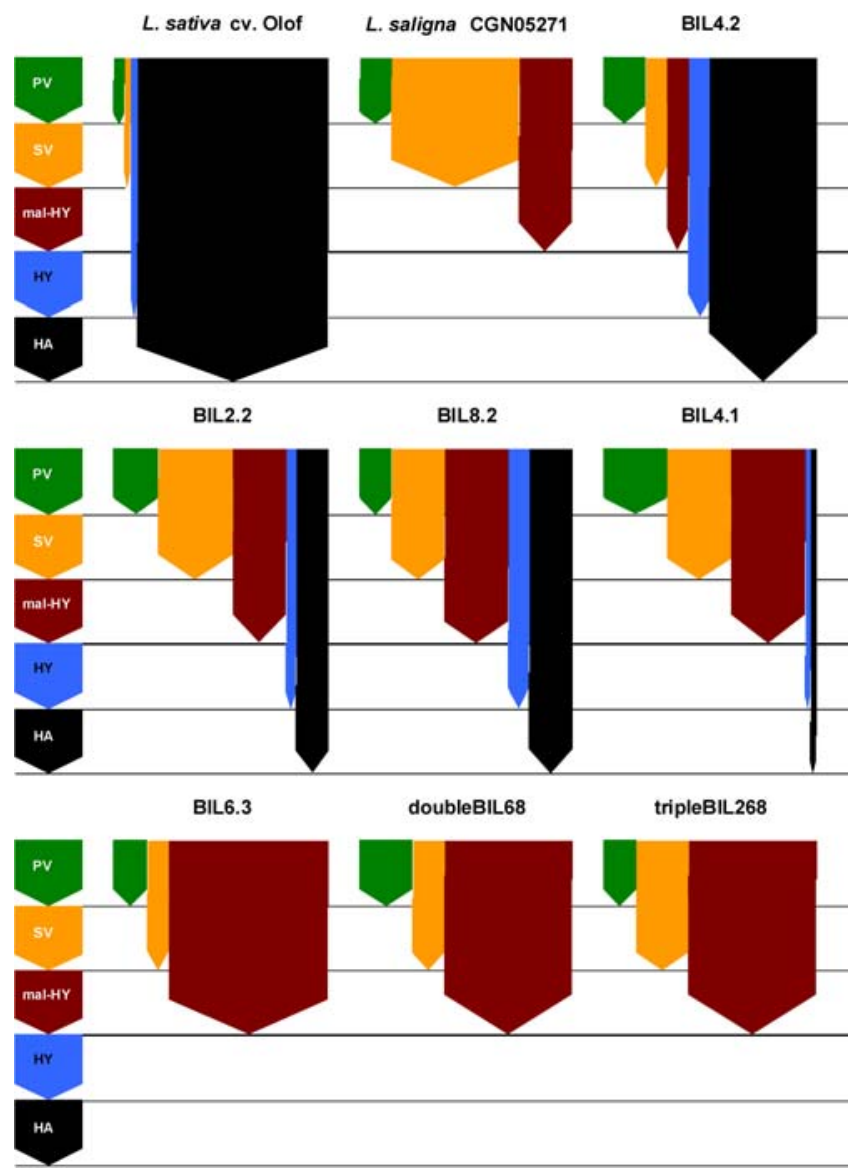

Fig. 3. Diagrammatic representation of the infection process of Bremia lactucae race Bl:14 in tested Lactuca genotypes. The barriers that B. lactucae may encounter while invading lettuce plants are represented on the left side with shaded arrows. $\mathrm{PV}=$ primary vesicle, $\mathrm{SV}=$ secondary vesicle, mal-HY = malformed hyphae like structure, $\mathrm{HY}=$ hyphae formation, and $\mathrm{HA}=$ haustorium formation. The widths of the arrows are proportional to the fractions of the infection units encountering the respective barriers.
BIL6.3, doubleBIL68, and tripleBIL268 plants all had a high proportion of IU that formed SV but no IU that formed healthy HY and HA. In these lines, the infection was completely arrested before HY formation. Large proportions (approximately $80 \%$ ) of mal-HY were observed in these three lines and most mal-HY were accompanied by SEHR (Table 2; Figs. 3 and 4). Such a prehyphal resistance response was similar to that of L. saligna; however, the pathogen development was obviously arrested earlier in $L$. saligna than in tripleBIL268, doubleBIL68, and BIL6.3. For BIL4.2, we observed only minor differences in pathogen development from that of L. sativa cv. Olof at 48 hpi (Table 2; Fig. 3).

\section{DISCUSSION}

The present study aims to determine how many L. saligna resistance alleles of QTL are sufficient to impart complete resistance to $B$. lactucae, whether they interact with each other or not, and to identify the microscopical resistance response underlying each target QTL. To achieve these objectives, we selected four target BIL with target QTL and developed 11 combiBIL in which $L$. saligna introgressions carrying QTL for resistance were combined in the L. sativa background. Disease tests and histological studies were carried out on the individual target BIL and combiBIL.

\section{QTL combinations lead to increased resistance.}

At both young and adult plant stages, the epistatic interactions of QTL $r b q 4$ (introgression 8.2), rbq5 (introgression 2.2), and $r b q 6+11$ (introgression 6.3+4.1) in combiBIL led to an increased level of resistance (decreased RIS) compared with their respective individual BIL. This decrease in RIS was 97 to $99 \%$ at the young plant stage and 54 to $90 \%$ at the adult plant stage $\left(\mathrm{ADT}_{\mathrm{G}}\right.$ and $\left.\mathrm{ADT}_{\mathrm{F}}\right)$, as compared with $L$. sativa cv. Olof (Fig. 2). Such effects even resulted in complete resistance of tripleBIL268 towards B. lactucae at young plant stage.

The resistance alleles of $r b q 7$ (introgression 4.2) in combiBIL did not contribute to increased resistance levels and only showed similar resistance levels as the individual BIL (Fig. 2).

At the seedling stage, $r b q 6+11$ and $r b q 7$, when combined, showed an epistatic interaction with a negative effect, since $r b q 7$ lost its resistance effect after being combined with rbq6+11. Similar situations of epistatic interactions of multiple loci with increased or decreased resistance levels were reported previously in other QTL pyramiding studies, for example, in barley to stripe rust (Castro et al. 2003a and b; Richardson et al. 2006) and in wheat (Triticum aestivum) to leaf rust caused by Puccinia recondita f. sp. tritici (Kloppers and Pretorius 1997).

Table 2. Development of Bremia lactucae race Bl:14 in the tested Lactuca genotypes at 48 h postinoculation ${ }^{\mathrm{a}}$

\begin{tabular}{|c|c|c|c|c|c|c|c|c|c|}
\hline \multirow[b]{2}{*}{ Genotype } & \multirow[b]{2}{*}{ RIS\% } & \multirow[b]{2}{*}{ No. PV (IU)/plant } & \multicolumn{4}{|c|}{ Frequency/proportion (\%) } & \multirow[b]{2}{*}{ HR\% } & \multirow[b]{2}{*}{ EHR\% } & \multirow[b]{2}{*}{ SEHR \% } \\
\hline & & & SV/PV & mal-HY/SV & HY/SV & HА/HY & & & \\
\hline Lactuca sativa & $100^{\mathrm{S}}$ & 81 & $97^{\mathrm{S}}$ & $0^{\mathrm{S}}$ & $94^{\mathrm{S}}$ & $94^{\mathrm{S}}$ & $56^{\mathrm{S}}$ & 98 & 2 \\
\hline L. saligna & $0.0^{\mathrm{O}}$ & 167 & $86^{\mathrm{O}}$ & $23^{\mathrm{O}}$ & $0^{\mathrm{O}}$ & $0^{\mathrm{O}}$ & $88^{\circ}$ & 97 & 3 \\
\hline BIL 4.4 & $119^{\mathrm{O}, \mathrm{S}}$ & 43 & $94^{\mathrm{S}}$ & $0^{S}$ & $94^{\mathrm{S}}$ & $96^{\mathrm{S}}$ & $43^{\mathrm{S}}$ & 100 & 0 \\
\hline BIL4.2 & $43^{\mathrm{O}, \mathrm{S}}$ & 28 & $87^{\circ}$ & 9 & $77^{\mathrm{O}, \mathrm{S}}$ & $79^{S}$ & 65 & 93 & 7 \\
\hline BIL2.2 & $15^{\mathrm{O}, \mathrm{S}}$ & 30 & $75^{\mathrm{O}, \mathrm{S}}$ & $24^{\mathrm{O}}$ & $15^{\mathrm{O}, \mathrm{S}}$ & $83^{S}$ & $88^{\mathrm{O}}$ & $33^{\mathrm{O}, \mathrm{S}}$ & $67^{\mathrm{O}, \mathrm{S}}$ \\
\hline BIL8.2 & $56^{\mathrm{O}, \mathrm{S}}$ & 32 & $86^{\mathrm{O}}$ & $35^{\mathrm{O}}$ & $29^{\mathrm{O}, \mathrm{S}}$ & $60^{\mathrm{O}, \mathrm{S}}$ & 68 & $47^{\mathrm{O}, \mathrm{S}}$ & $53^{\mathrm{O}, \mathrm{S}}$ \\
\hline BIL4.1 & $37^{\mathrm{O}, \mathrm{S}}$ & 39 & $69^{\mathrm{O}, \mathrm{s}}$ & $49^{\mathrm{O}}$ & $7^{\mathrm{O}}$ & $60^{\mathrm{O}, \mathrm{S}}$ & $56^{\mathrm{S}}$ & $32^{\mathrm{O}, \mathrm{S}}$ & $68^{\mathrm{O}, \mathrm{S}}$ \\
\hline BIL6.3 & $19^{\mathrm{O}, \mathrm{S}}$ & 66 & $83^{\mathrm{O}}$ & $88^{\mathrm{O}, \mathrm{S}}$ & $0^{\mathrm{O}}$ & $0^{\mathrm{O}}$ & 64 & $19^{\mathrm{O}, \mathrm{S}}$ & $81^{\mathrm{O}, \mathrm{S}}$ \\
\hline doubleBIL68 & $0.3^{\mathrm{O}}$ & 77 & $73^{\mathrm{O}, \mathrm{S}}$ & $82^{\mathrm{O}, \mathrm{S}}$ & $0^{\mathrm{O}}$ & $0^{\mathrm{O}}$ & $95^{\circ}$ & $19^{\mathrm{O}, \mathrm{S}}$ & $81^{\mathrm{O}, \mathrm{S}}$ \\
\hline tripleBIL268 & $0.0^{\mathrm{O}}$ & 129 & $83^{\mathrm{O}}$ & $74^{\mathrm{O}, \mathrm{S}}$ & $0^{\mathrm{O}}$ & $0^{\mathrm{O}}$ & $84^{\mathrm{O}}$ & $15^{\mathrm{O}, \mathrm{S}}$ & $85^{\mathrm{O}, \mathrm{S}}$ \\
\hline
\end{tabular}

${ }^{a}$ BIL = backcross inbred lines; RIS = relative infection severity compared with $L$. sativa cv. Olof at 10 days postinoculation; PV = primary vesicle; IU = infection unit; SV = secondary vesicle; mal-HY = malformed hyphae (HY)-like structure; HA = haustorium; HR\% = percentage of IU associated with hypersensitive response (HR); EHR\% = percentage of HR-associated IU in which HR was limited to epidermis; SEHR $\%=$ percentage of HR-associated IU in which HR was extended to subepidermal-HR. The absolute infection severity of cv. Olof was $84 \%$. Superscript letters o and s indicate significant difference from L. sativa (cv. Olof) and L. saligna (CGN05271), respectively. 
The complete and almost complete resistance of, tripleBIL268, quatroBIL2468, and doubleBIL68 suggests a redundancy of QTL in the nonhost resistance of $L$. saligna, in which 15 QTL for resistance have been identified. So far, we only have evidence for the almost complete to complete resistance of doubleBIL68, tripleBIL268, and quatroBIL2468 at the young plant stage, but we expect that these three lines will also show very high levels of resistance at the adult plant stage. At the seedling stage, tripleBIL268 might not be more resistant than doubleBIL28, since BIL6.3, BIL8.2, and doubleBIL68 were all susceptible (Zhang et al. in press), but the resistance level of quatroBIL2468 is more difficult to predict, as more interactions between the QTL may occur. TripleBIL268 may need one or more QTL to be resistant at other developmental stages. We hypothesize that the abundant QTL of $L$. saligna might be a good backup in case some QTL are overcome by B. lactucae races.

\section{Microscopical resistance responses conferred by the QTL.}

We observed that the nonhost resistance of $L$. saligna CGN05271 was mainly due to a prehyphal resistance, which was in agreement with previous findings based on the inoculation of L. saligna accessions with B. lactucae races B1:5 and Bl:16 on detached tissues (Lebeda and Reinink 1994; Sedlářová et al. 2001). Compared with those previous results, our samples showed generally higher HR (HR\%) in all tested lines, even in the susceptible reference lines. This could be due to differences in growth conditions, tissue types, or staining method used between studies.
The microscopical resistance responses in BIL2.2, BIL8.2, and BIL4.1 might be characterized as incomplete prehyphal resistance associated with SEHR (Table 2; Figs. 3 and 4). For BIL6.3 $(r b q 6+11)$, doubleBIL68 $(r b q 4+r b q 6+11)$, and tripleBIL268 $(r b q 4+r b q 5+r b q 6+11)$, the resistance response might be characterized as complete prehyphal resistance associated with SEHR. This resistance response most resembled that in $L$. saligna. However, the pathogen could form HA during the later phase of infection in BIL6.3 and doubleBIL68, else we would not have observed 16 and $0.3 \%$ RIS in BIL6.3 and doubleBIL68, respectively (Table 2). DoubleBIL68 and tripleBIL268 showed a similar resistance response as that in BIL6.3 but different from that of BIL8.2 and BIL2.2 (Fig. 3). This indicates that the effect of $r b q 6+11$ in BIL6.3 masks most of the effect from rbq4 in BIL8.2 and $r b q 5$ in BIL2.2 (Table 2; Fig. 3).

BIL4.2 ( $r b q 7)$ delays defensive action until after 48 hpi compared with other QTL lines, since before $48 \mathrm{hpi}$, the infection was nearly as successful as in the susceptible cv. Olof, but infection severity (RIS) was significantly reduced (Table 2). Such a delayed defensive action might be redundant with the earlier acting resistance responses of $r b q 6+11, r b q 4$, and $r b q 5$ and therefore explain why the resistance level did not increase when it was combined with other QTL.

Interestingly, the resistance responses of tripbleBIL268 do not completely resemble that of $L$. saligna CGN05271, although this line showed complete resistance. The SEHR-based resistance in doubleBIL68 and tripleBIL268 were derived from

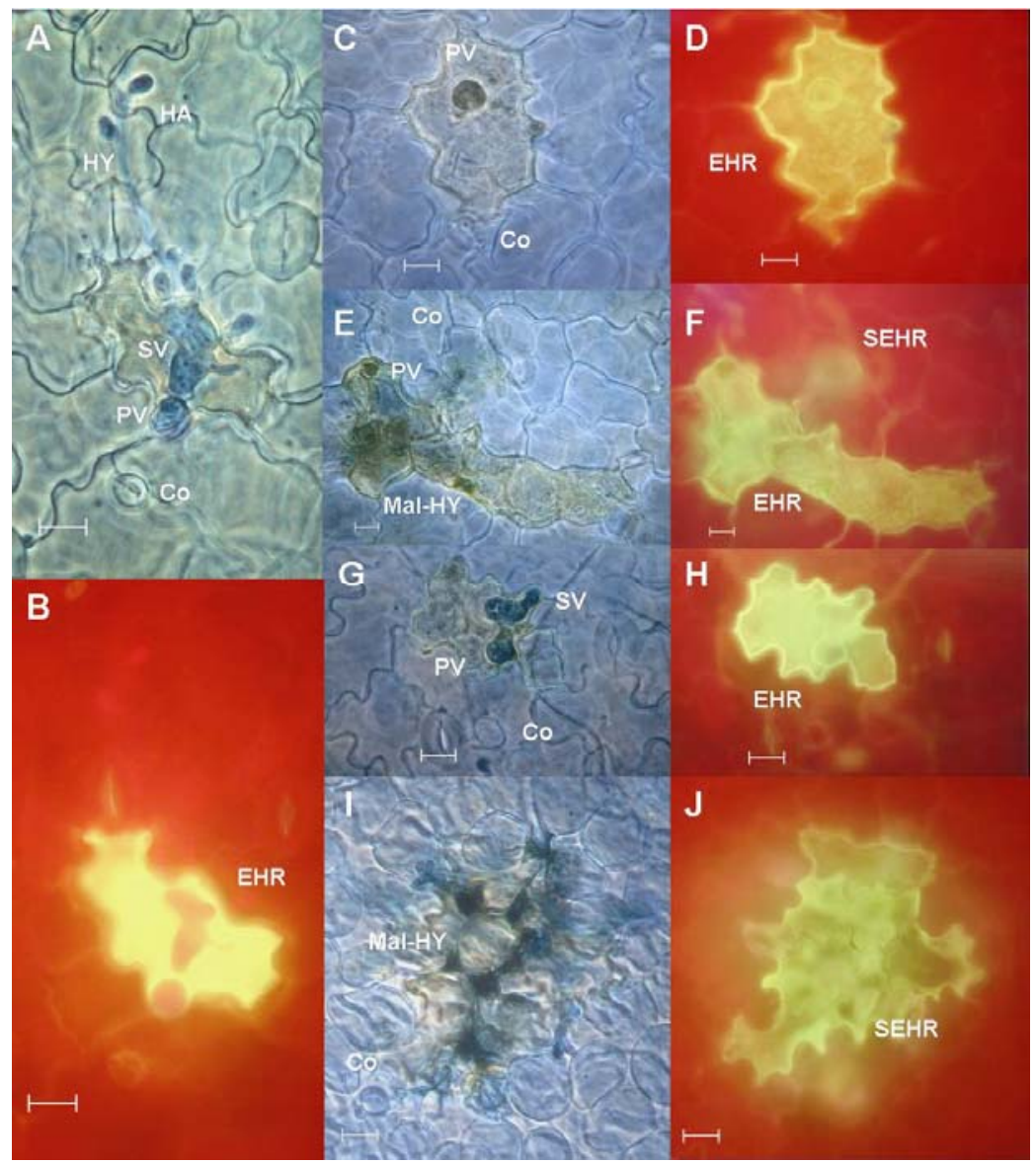

Fig. 4. Microscopic illustration of Bremia lactucae race Bl:14 development stages observed in tested Lactuca genotypes. The scale bar at the left lower corner $=20 \mu \mathrm{m} . \mathrm{Co}=$ conidium, $\mathrm{PV}=$ primary vesicle, $\mathrm{SV}=$ secondary vesicle, $\mathrm{HY}=$ hyphae, $\mathrm{HA}=$ haustorium, EHR $=$ epidermal hypersensitive response (HR) cell, SEHR = subepidermal HR cell. A and B, Infection units (IU) in L. sativa cv. Olof with hypha and haustoria and HR epidermal cell; $\mathbf{C}$ and $\mathbf{D}$, IU in L. saligna CGN05271 with PV and HR epidermal cell; $\mathbf{E}$ and F, IU in L. saligna CGN05271 with deformed HY-like structure and HR epidermal cell; G and $\mathbf{H}$, IU in BIL2.2 with SV and HR epidermal cell; and $\mathbf{I}$ and $\mathbf{J}$, IU in doubleBIL68 with deformed HY-like structure and EHR and SEHR cells. 
BIL2.2, BIL6.3, and BIL8.2 and was not observed in $L$. saligna CGN05271 and L. sativa cv. Olof. The reasons for such deviation between responses of dissected resistance and of the intact nonhost resistance remain unknown. One possible explanation may be that, in the nonhost $L$. saligna, the resistance level is very high and the pathogen is inhibited early, before it can induce SEHR. In the BIL and combiBIL, resistance is less strong, hence the inhibition might be either slightly later, weaker, or both, allowing the pathogen to more greatly affect the tissue and, hence, induce $H R$ at a later phase in subepidermal cells. In the compatible interaction with either lower doses of QTL or none at all, the pathogen organizes its infection well and suppresses HR more than in the nonhost parent.

Observations at timepoints later than 48 hpi may be useful for further characterizing the resistance responses conferred by these QTL. In the future, histological studies may assist in determining of the QTL effect and facilitate both the fine mapping of QTL and ongoing QTL cloning.

\section{Perspectives for breeding.}

Pyramiding or stacking QTL for resistance by MAS has been suggested as an effective strategy for increasing the level of probably durable resistance towards different pathogens or pests (Richardson et al. 2006; Varshney et al. 2006; Yang et al. 2005).

Our four target resistance QTL are recessive and most probably not specific to individual $B$. lactucae races (Jeuken et al. 2008; Zhang et al. in press). We pyramided these four target QTL in 11 combiBIL. The most resistant combiBIL were doubleBIL68 and tripleBIL268, as they showed very low or no infection level by B. lactucae (Fig. 2).

QTL in BIL2.2 (rbq5), BIL6.3 $(r b q 6+11)$, and BIL8.2 $(r b q 4)$ are more interesting for introgression in lettuce cultivars than the QTL in BIL4.2 ( $r b q 7)$ for three reasons: i) the former three QTL showed epistatic interactions with increased resistance levels, while $r b q 7$ did not, ii) the microscopical resistance responses of these three QTL acted at an early phase of pathogen development compared with $r b q 7$, and iii) the former three BIL have a rather normal lettuce cultivar morphology, while BIL4.2 showed undesired traits (Jeuken and Lindhout 2004). All the above features of the QTL $r b q 4$, rbq5, and $r b q 6+11$ suggest that introducing them into a lettuce cultivar might lead to sufficient and durable resistance to B. lactucae.

\section{MATERIALS AND METHODS}

\section{Plant materials.}

For QTL pyramiding, four BIL harboring quantitative resistance alleles of QTL to B. lactucae were used, namely, BIL2.2 (rbq5), BIL4.2 (rbq7), BIL6.3 (rbq6+11), and BIL8.2 (rbq4). The nomenclature of the BIL refers to the location of the introgression. For example, BIL2.2 stands for the second introgression on chromosome 2 (Jeuken and Lindhout 2004). The chromosome numbering is according to the L. saligna $\times$ L. sativa genetic map by Jeuken and associates (2001) and the integrated lettuce genetic map by Truco and associates (2007), except for chromosomes 3,6 , and 8 , which are numbered 6,8 , and 3 in the integrated lettuce genetic map. Each BIL contains one homozygous L. saligna CGN05271 (nonhost) introgression in L. sativa cv. Olof (host) background, except BIL6.3, which contains an additional introgression at the top of chromosome 4 from 0 to $32 \mathrm{cM}$, harboring Rbq11. The resistance alleles of QTL on introgressions 2.2, 4.2, 6.3, and 8.2 are effective at both the young and adult plant (greenhouse) stages, and BIL2.2 and BIL4.2 were also effective at the seedling stage.
The following genotypes were included in the disease tests to evaluate the resistance level of each combiBIL (lines containing more than one target QTL): i) the 11 combiBIL that were developed in the present study, including six doubleBIL, four tripleBIL, and one quatroBIL; ii) the individual BIL with QTL, BIL2.2, BIL4.2, BIL6.3 and BIL8.2; iii) the parental lines L. saligna CGN5271 (nonhost) and L. sativa cv. Olof (host); and iv) three additional susceptible reference lines, BIL4.4, BIL5.1, and BIL8.1 (Zhang et al. in press).

\section{Pyramiding QTL procedure.}

According to the formula $C(n, m)=n ! /(m ! \times(n-m) !(C=$ number of combinations; $m=$ number of QTL in one line; $n=$ total number of QTL), 11 combiBIL should be developed from four target BIL to obtain all possible QTL combinations. We first crossed BIL2.2 with BIL6.3 and BIL4.2 with BIL8.2. The $F_{1}$ progeny from these two crosses were selfed to obtain $F_{2}$ plants. Among these $\mathrm{F}_{2}$ plants, we selected doubleBIL2.2+6.3 and doubleBIL4.2+8.2, respectively, MAS. We then crossed doubleBIL2.2+6.3 with doubleBIL4 $2+8.2$, followed by one round of selfing to obtain a second $F_{2}$ population. This second $\mathrm{F}_{2}$ population and subsequent generations from selfing $\left(\mathrm{F}_{3}\right.$ and $\mathrm{F}_{4}$ ) were genotyped and selection was carried out for the other combiBIL (Fig. 1). The combiBIL are designated by the chromosome numbers in which the introgression is located, For example, we refer to the doubleBIL2.2+6.3 as "doubleBIL26" and tripleBIL2.2+6.3+8.2 as "tripleBIL268" (Fig. 2).

\section{Genotyping.}

First, the $\mathrm{F}_{2}$ plants were genotyped by introgression-specific EST markers that map to BIL2.2, BIL4.2, BIL6.3, and BIL8.2 introgressions. The positions and primers of these markers were described by Jeuken and associates (2008). To confirm that the complete introgression was present, selected plants were again genotyped using genome-wide amplified fragment length polymorphism (AFLP) analyses (Jeuken et al. 2001). DNA isolation for the first screening using EST markers was done by the $\mathrm{NaOH}$ method (Wang et al. 1993). DNA isolation for confirmation and genome-wide genotyping by AFLP was performed according to the modified CTAB method (Jeuken et al. 2001; Stewart and Via 1993). The amplification of EST markers, cleaved amplified polymorphic sequence digestion, and AFLP analysis were described previously (Jeuken et al. 2008). Each final candidate combiBIL plant was genotyped independently at least three times.

\section{Disease evaluation.}

To evaluate the infection severities of combiBIL and reference lines, we performed disease tests at the seedling, young plant, and adult plant stages. The experimental procedures of the SDT, YDT, $\mathrm{ADT}_{\mathrm{G}}$, and $\mathrm{ADT}_{\mathrm{F}}$ were described previously (Jeuken et al. 2008; Zhang et al. in press).

We first tested six doubleBIL, together with the individual BIL, the parental lines, and the reference lines using four different types of bioassays. For SDT, YDT, and $\mathrm{ADT}_{\mathrm{G}}$, two independent tests were performed, one with $B$. lactucae race $\mathrm{Bl}: 14$ and another with race $\mathrm{Bl}: 16$. Inoculum concentrations used in these tests were $2 \times 10^{5}$ to $4 \times 10^{5}$ conidia/ml. For $\mathrm{ADT}_{\mathrm{F}}$, we performed artificial inoculation by spraying with $\mathrm{Bl}: 14$ at a concentration of $1 \times 10^{4}$ conidia/ml. The virulence spectra of races $\mathrm{Bl}: 14$ and $\mathrm{Bl}: 16$, pathogen maintenance, inoculum preparation, and the method of inoculation were as described previously (Jeuken and Lindhout 2002).

For the SDT, we tested 16 seedlings per line in a climate chamber. The seedlings were 12 days old, and the detached cotyledons were then inoculated with the inoculum. For the YDT, we tested six young plants (3 to 4 weeks old) per geno- 
type, under the same conditions as those used for the SDT. For the $\mathrm{ADT}_{\mathrm{G}}$, seven plants ( 8 weeks old) were used per genotype per experiment with four detached leaf disks per plant. In all three bioassay types, we evaluated infection severities from 8 to 10 days postinoculation by estimating the percentage of sporulating area per cotyledon, representative leaf, and leaf disk. The representative leaves are two of the youngest, wellexpanded leaves at the moment of inoculation of each plant.

The $\mathrm{ADT}_{\mathrm{F}}$ was carried out in sandy soil at Wageningen in The Netherlands in the autumn of 2006 according to a randomized, complete block design with five replicates. Each plot contained five rows of five plants from one genotype and interplant spacing of $30 \mathrm{~cm}$. One month after transplantation, the plants were artificially inoculated with $B$. lactucae race $\mathrm{Bl}: 14$. Disease evaluations were done at 20 days after inoculation. We performed a semiquantitative assessment based on observations per plot with a scale ranging from 0 (completely resistant) to 10 (the most susceptible), as described by Zhang and associates (in press).

In subsequent experiments, we evaluated all 11 combiBIL, four individual BIL, the parental lines, and the reference lines using three additional YDT, by inoculation with race Bl:14 at a similar inoculum concentration as for previous YDT on doubleBIL. The YDT is the most efficient test in terms of time and space and is relevant, since the level of resistance is positively correlated with the level of resistance in adult plants (Zhang et al. in press).

All the severity percentage data were arcsine square roottransformed to improve the homogeneity of the residual variance. A two-way analysis of variance was performed on the $\mathrm{ADT}_{\mathrm{F}}$ data and the linear mixed model was employed to analyze the SDT, YDT, and $\mathrm{ADT}_{\mathrm{G}}$ data. We set "genotype" and "inoculation box" as fixed factors and "plant" as the random factor. The results are presented as the RIS for each examined line, which was calculated using the formula RIS $=(b / a) \times$ $100 \%$, in which $a$ and $b$ are the absolute infection severity percentages of $L$. sativa cv. Olof and the examined line, respectively. Hence, the severity of L. sativa cv. Olof was always $100 \%$ in different bioassay types.

The Tukey honestly significant differences test $(\alpha=0.05)$ was used for multiple comparisons between all the genotypes within one type of disease test. The criterion for detecting an introgression with an effect on the infection level was set, using a significant difference from the susceptible parental line L. sativa cv. Olof. We considered introgressions that were associated with significantly higher or lower infection levels than $L$. sativa cv. Olof to harbor a QTL allele for resistance or susceptibility. To detect epistatic interactions of the QTL, we set a significance level of 0.05 for the multiple comparisons between the individual BIL and the combiBIL in a pairwise manner. All statistical analyses were performed with SPSS12.0.1 (SPSS Inc., Chicago).

\section{Histology.}

An additional YDT was performed for a histological study on lines including L. sativa cv. Olof, L. saligna CGN05271, BIL4.4, BIL2.2, BIL4.2, BIL6.3, BIL8.2, BIL4.1, one doubleBIL that showed epistatic interaction with positive effects between the two combined QTL (doubleBIL68), and one tripleBIL that showed complete resistance to $B$. lactucae at the young plant stage (tripleBIL268). The other combiBIL were not included, since they did not display higher resistance levels than doubleBIL68 and tripleBIL268. Six 4-week-old plants of each genotype were inoculated with $B$. lactucae race Bl:14 conidium suspension $\left(3 \times 10^{5}\right.$ conidia/ml $)$. For each genotype, three plants were used for microscopic observations and the remaining three for macroscopic observations (the infection severity evaluation and data analysis were as described for YDT).

For microscopic observations, two leaf segments, $1 \times 2 \mathrm{~cm}^{2}$ in size, per plant were sampled at $48 \mathrm{hpi}$. The sampled leaf segments were first fixed in an acetic acid and ethanol (1:3, $\mathrm{vol} / \mathrm{vol})$ solution and were stained with lacto-phenol and trypan blue as described by Van Damme and associates (2005). The leaf segments were examined with a phase contrast (Zeiss Axiophot) microscope equipped with a digital camera at $400 \times$ magnification. Photographs were taken by the digital camera and transferred to a computer using an AxioVison LE Rel.4.6 (Carl Zeiss, Oberkochen, Germany).

The typical compatible infection process of B. lactucae starts from conidium germination, followed by formation of an appressorium and penetration of the epidermal cell wall, then by formation of a PV, a SV, HY, and HA within 24 hpi (Ingram et al. 1976; Lebeda and Reinink 1994).

On each leaf segment, we counted the total number of IU and recorded the presence of the various infection structures of B. lactucae. We defined an IU as a germinated conidium with a PV. Except for the PV, the frequency of subsequent infection structures was calculated as a proportion (percentages) of the structures from which they were derived. To identify the HR, autofluorescence of trypan blue-stained tissue was observed as described by Vleeshouwers and associates (2000). The number of IU that were associated with EHR or SEHR cells, or both, was recorded. HR frequency (Table 2; HR\% column) was calculated using the number of IU that showed HR (EHR and SEHR) at infection sites divided by the total number of IU.

For data analysis, we combined the number of IU from two leaf segments of the same plant to obtain a better representation of pathogen development in that plant. The frequency of each infection structure was compared with that on $L$. saligna CGN05271 and L. sativa cv. Olof, using the Mann-Whitney U test at the $95 \%$ level. The statistical analyses were performed with SPSS12.0.1.

For L. sativa cv. Olof, L. saligna CGN05271, BIL4.4, BIL2.2, and BIL4.2, histological observations were also carried out in a similar way but in a separate experiment. The results were similar between the previous and the present histological test. Here, we only show the results from the present and most extensive study.

\section{ACKNOWLEDGMENTS}

The authors acknowledge support by the Dutch Technology Foundation STW, Applied Science Division of The Netherlands Organization for Scientific Research (NWO), and the Technology Program of the Ministry of Economic Affairs. EST data was obtained from The Compositae Genome Project website, which was supported by the United States Department of Agriculture Initiative for Future Agriculture and Food Systems and the National Science Foundation Plant Genome programs. We also thank C. Maliepaard for the suggestions on the statistical analysis.

\section{LITERATURE CITED}

Atienza, S. G., Jafary, H., and Niks, R. E. 2004. Accumulation of genes for susceptibility to rust fungi for which barley is nearly a nonhost results in two barley lines with extreme multiple susceptibility. Planta 220:71-79.

Bennett, M., Gallagher, M., Fagg, J., Bestwick, C., Paul, T., Beale, M., and Mansfield, J. 1996. The hypersensitive reaction, membrane damage and accumulation of autofluorescent phenolics in lettuce cells challenged by Bremia lactucae. Plant J. 9:851-865.

Bestwick, C. S., Adam, A. L., Puri, N., and Mansfield, J. W. 2001. Characterisation of and changes to pro- and anti-oxidant enzyme activities during the hypersensitive reaction in lettuce (Lactuca sativa L.). Plant Sci. 161:497-506.

Castro, A. J., Chen, X. M., Corey, A., Filichkina, T., Hayes, P. M., Mundt, C., Richardson, K., Sandoval-Islas, S., and Vivar, H. 2003a. Pyramiding and validation of quantitative trait locus (QTL) alleles determining re- 
sistance to barley stripe rust: Effects on adult plant resistance. Crop Sci. 43:2234-2239.

Castro, A. J., Chen, X. M., Hayes, P. M., and Johnston, M. 2003b. Pyramiding quantitative trait locus (QTL) alleles determining resistance to barley stripe rust: Effects on resistance at the seedling stage. Crop Sci. 43:651-659.

Ferreira, R. B., Monteiro, S., Freitas, R., Santos, C. N., Chen, Z. J., Batista, L. M., Duarte, J., Borges, A., and Teixeira, A. R. 2006. Fungal pathogens: The battle for plant infection. Crit. Rev. Plant Sci. 25:505-524.

Heath, M. C. 1981. A generalized concept of host-parasite specificity. Phytopathology 71:1121-1123.

Heath, M. C. 2000. Nonhost resistance and nonspecific plant defenses. Curr. Opin. Plant Biol. 3:315-319.

Heath, M. C. 2001. Non-host resistance to plant pathogens: Nonspecific defense or the result of specific recognition events? Physiol. Mol. Plant Pathol. 58:53-54.

Ingram, D. S., Sargent, J. A., and Tommerup, I. C. 1976. Structural aspects of infection of lettuce by biotrophic fungi Bremia lactucae. Pages 43-78 in: Biochemical aspects of Plant Parasite Relationships. J. Friend and D. R. Threlfall, eds. Academic Press, London.

Jeuken, M., and Lindhout, P. 2002. Lactuca saligna, a non-host for lettuce downy mildew (Bremia lactucae), harbors a new race-specific Dm gene and three QTLs for resistance. Theor. Appl. Genet. 105:384-391.

Jeuken, M. J. W., and Lindhout, P. 2004. The development of lettuce backcross inbred lines (BILs) for exploitation of the Lactuca saligna (wild lettuce) germplasm. Theor. Appl. Genet. 109:394-401.

Jeuken, M., van Wijk, R., Peleman, J., and Lindhout, P. 2001. An integrated interspecific AFLP map of lettuce (Lactuca) based on two L. sativa $\times$ L. saligna $\mathrm{F}_{2}$ populations. Theor. Appl. Genet. 103:638-647.

Jeuken, M. J. W., Pelgrom, K., Stam, P., and Lindhout, P. 2008. Efficient QTL detection for nonhost resistance in wild lettuce: Backcross inbred lines versus $F_{2}$ population. Theor. Appl. Genet. 116:845-857.

Kamoun, S., Huitema, E., and Vleeshouwers, V. 1999. Resistance to oomycetes: A general role for the hypersensitive response? Trends Plant Sci. 4:196-200.

Kloppers, F. J., and Pretorius, Z. A. 1997. Effects of combinations amongst genes $\operatorname{Lr} 13, \operatorname{Lr} 34$ and $\operatorname{Lr} 37$ on components of resistance in wheat to leaf rust. Plant Pathol. 46:737-750.

Lebeda, A., Pink, D., and Astley, D. 2002. Aspects of the interactions between wild Lactuca spp. and related genera and lettuce downy mildew ( Bremia lactucae ). Pages 85-117. In: Advances in Downy Mildew Research. P. T. N. Spencer-Phillips, U. Gisi, and A. Lebeda, eds. Kluwer Academic Publishers, Dordrecht, The Netherlands.

Lebeda, A., Pink, D. A. C., and Mieslerova, B. 2001. Host-parasite specificity and defense variability in the Lactuca spp.-Bremia lactucae pathosystem. J. Plant Pathol. 83:25-35.

Lebeda, A., and Reinink, K. 1994. Histological characterization of resistance in Lactuca saligna to lettuce downy mildew (Bremia lactucae). Physiol. Mol. Plant Pathol. 44:125-139.

Lebeda, A., and Schwinn, F. J. 1994. The downy mildews: An overview of recent research progress. J. Plant Dis. Prot. 101:225-254.

Lebeda, A., Sedlářová, M., Lynn, J., and Pink, D. 2006. Phenotypic and histological expression of different genetic backgrounds in interactions between lettuce, wild Lactuca spp., L. sativa $\times$ L. serriola hybrids and Bremia lactucae. European J. Plant Pathol. 115:431-441.
Maclean, D. J., and Tommerup, I. C. 1979. Histology and physiology of compatibility and incompatibility between lettuce and the downy mildew fungus, Bremia Lactucae Regel. Physiol. Plant Pathol. 14:291-312.

Mysore, K. S., and Ryu, C. M. 2004. Nonhost resistance: How much do we know? Trends Plant Sci. 9:97-104.

Niks, R. E. 1988. Nonhost plant species as donors for resistance to pathogens with narrow host range 2 . Concepts and evidence on the genetic basis of nonhost resistance. Euphytica 37:89-99.

Niks, R. E., and Dekens, R. G. 1991. Prehaustorial and posthaustorial resistance to wheat leaf rust in diploid wheat seedlings. Phytopathology 81:847-851.

Niks, R. E., and Rubiales, D. 2002. Potentially durable resistance mechanisms in plants to specialised fungal pathogens. Euphytica 124:201216.

Richardson, K. L., Vales, M. I., Kling, J. G., Mundt, C. C., and Hayes, P. M. 2006. Pyramiding and dissecting disease resistance QTL to barley stripe rust. Theor. Appl. Genet. 113:485-495.

Schweizer, P. 2007. Nonhost resistance of plants to powdery mildew-New opportunities to unravel the mystery. Physiol. Mol. Plant Pathol. 70:3-7.

Sedlářová, M., Lebeda, A., and Pink, D. A. C. 2001. The early stages of interaction between effective and non-effective race-specific genes in Lactuca sativa, wild Lactuca spp. Bremia lactucae (race NL16). J. Plant Dis. Protect. 108:477-489.

Stewart, C. N., and Via, L. E. 1993. A rapid CTAB DNA isolation technique useful for rapid fingerprinting and other PCR applications. Biotechniques 14:748-750.

Truco, M. J., Antonise, R., Lavelle, D., Ochoa, O., Kozik, A., Witsenboer, H., Fort, S. B., Jeuken, M. J. W., Kesseli, R. V., Lindhout, P., Michelmore, R. W., and Peleman, J. 2007. A high-density, integrated genetic linkage map of lettuce (Lactuca spp.). Theor. Appl. Genet. 15:735-746.

Van Damme, M., Andel, A., Huibers, R. P., Panstruga, R., Weisbeek, P. J., and Van den Ackerveken, G. 2005. Identification of Arabidopsis loci required for susceptibility to the downy mildew pathogen Hyaloperonospora parasitica. Mol. Plant-Microbe Interact. 18:583-592.

Varshney, R. K., Hoisington, D. A., and Tyagi, A. K. 2006. Advances in cereal genomics and applications in crop breeding. Trends Biotechnol. 24:490-499.

Vleeshouwers, V. G. A. A., van Dooijeweert, W., Govers, F., Kamoun, S., and Colon, L. T. 2000. The hypersensitive response is associated with host and nonhost resistance to Phytophthora infestans. Planta 210:853864.

Wang, H., Qi, M. Q., and Cutler, A. J. 1993. A simple method of preparing plant samples for PCR. Nucleic Acids Res. 21:4153-4154.

Yang, Z. P., Gilbert, J., Fedak, G., and Somers, D. J. 2005. Genetic characterization of QTL associated with resistance to Fusarium head blight in a doubled-haploid spring wheat population. Genome 48:187-196.

Zhang, N. W., Lindhout, P., Niks, R. E., and Jeuken, M. J. W. Genetic dissection of Lactuca saligna nonhost resistance to downy mildew at various lettuce developmental stages. Plant Pathol. In press.

\section{AUTHOR-RECOMMENDED INTERNET RESOURCE}

The Compositae Genome Project website: compgenomics.ucdavis.edu 